\title{
FURTHER PROBLEMS CONCERNING FINITE GEOMETRIES AND FINITE GROUPS
}

\author{
WILLIAM M. KANTOR
}

This note is intended as a very long footnote to the other finite geometry papers in this volume. Areas of overlap between finite geometry (or combinatorics) and finite groups will be listed which were either not mentioned, or were only briefly alluded to, at the conference. However, the descriptions and bibliography given here will also be brief: the goal is to indicate research directions, not to give a comprehensive survey.

1. Buekenhout and Tits discussed building-like geometries at this conference. Tits pointed out that they are readily constructed from arithmetic groups. However, flag-transitive examples may be harder to find, and may be of interest for arithmetic groups when their diagrams are extended Dynkin diagrams.

The only known finite flag-transitive examples having such diagrams are related to sporadic groups: one example of

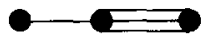

arising from the Lyons-Sims group, three of

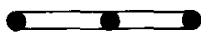

arising from $P \Omega^{-}(6,3) \cdot 2$, PSU $(6,2)$ and $\Omega^{+}(8,2)$ which are related to Fischer groups, and a fourth with the latter diagram (due to Ronan and Smith) arising from Suz. The universal covers of the corresponding complexes are buildings (by a theorem of Tits), and deserve study.

Further interesting examples of building-like geometries must exist. Presumably, finite examples even exist (undoubtedly with small groups) involving nonclassical generalized polygons.

2. The study of generalized polygons has recently attracted a great deal of attention. In addition to Tits' and Weiss' work on Moufang polygons and their generalizations (discussed by Weiss at the conference), there have been major

1980 Mathematics Subject Classification. Primary 20B25.

- American Mathematical Society 1980 
and elegantly geometric characterizations of the generalized quadrangles [34] and hexagons [40], [26] which arise from Chevalley groups. As yet, ${ }^{2} F_{4}(q)$ octagons have not been characterized geometrically.

However, no finite hexagons or octagons are presently known other than those arising from Chevalley groups. The existence of other examples is a fundamental problem in this area.

Only a few types of nonclassical generalized quadrangles are known, as well. Most of the known ones can be obtained by modifying a parabolic subgroup of a rank 2 Chevalley group. The elementary group-theoretic construction is found in [22], where new quadrangles are then obtained using $G_{2}(q)$. Analogous constructions exist for hexagons and octagons, but have yet to yield new examples. Entirely new construction procedures are needed; however, it seems very likely that these would also be group-theoretic.

In general, finite quadrangles seem tighter (and hence scarcer) than projective planes, hexagons much tighter still, and octagons so tight that very few types should exist. However, no Bruck-Ryser theorem is known. If a generalized polygon has $s+1$ points per line and $t+1$ lines per point (where $s>1$ and $t>1$ ), then some restrictions on $s$ and $t$ were obtained by Feit and Higman [14]. For quadrangles and octagons, the inequality $s \leqslant t^{2}$ has been known for a while [16], [17]. This year, $s \leqslant t^{3}$ was finally proved for hexagons [15]. Much more should be possible (compare [33, §4]).

A curious variation of these inequalities has recently been suggested. Cameron found a short combinatorial proof that a generalized quadrangle with 3 lines per point is necessarily finite, at the same time obtaining $s \leqslant t^{2}(=4)$ in this case. I obtained the same results when there are just 4 lines per point, by a group-theoretic argument.

Generalized polygons were a crucial ingredient in the determination of all 2-transitive collineation groups of finite projective spaces [6]. Embeddings of generalized quadrangles into projective spaces were classified in [3]. The corresponding problem for hexagons remains open. However, very restrictive embeddings of generalized polygons into projective spaces were essential in [6] (and led, incidentally, to an elementary construction of the $G_{2}(q)$ hexagons).

3. There is undoubtedly a great deal left to be learned about the geometry of finite Chevalley groups, even in the case of rank $\geqslant 3$. This was already made clear by Tits 20 years ago [35], [36], and was discussed in detail by Shult at the conference.

Geometry permeates recent work on groups generated by long root elements [8], [9], [20], as well as applications of that work [7], [21]. An entirely geometric approach was used in a different generation question [37]: the determination of all primitive subgroups of $\operatorname{GL}(n, q)$ generated by reflections.

4. The characters of the centralizer algebras are known for the permutation representations of $\operatorname{PSL}(n, q)$ on $i$-spaces for $1 \leqslant i \leqslant n-1$ [13], and of classical groups acting on maximal totally isotropic (or singular) subspaces [31]. These computations are highly geometric. The character values are polynomials in $q$, 
which are related to classical hypergeometric functions. Combinatorial applications of these results are given in [32]. Further results can be obtained using Hoefsmit's work [18] on the centralizer algebra of $1_{B}^{G}$.

The combinatorial analogues of permutation representations are coherent configurations, and in particular, association schemes. The latter have proven to be fundamental in coding theory [12], [28], and have motivated the study of the characters of especially important examples (as in the preceding paragraph).

A survey of many group theoretic uses of coherent configurations is given in [4].

More recently, coherent configurations have led in other directions. Some were discussed by Bannai at the conference. Another involves the Krein Condition and its combinatorial consequences, as well as properties of irreducible constituents of permutation characters [6]. Yet another generalizes the graph theoretic notion of coherent configuration to higher dimensional complexes [10].

5. Group theory has had important applications to coding theory (cf. [25]). The most familiar examples of this involve the Mathieu groups and the Golay codes. The relevance of invariant theory to coding theory is discussed in [25] and [29]. (Further combinatorial applications of invariant theory are described in [30] and its references.)

Quadratic residue codes arise by taking the degree $\frac{1}{2}(q+1)$ representations of $\operatorname{SL}(2, q)$ modulo a prime not dividing $q$. This led Ward [38] to study analogous codes arising from Weil representations of $\operatorname{Sp}(2 n, q)$.

Groups generated by transvections over $\mathrm{GF}(2)$ have very recently been crucial in the study (by Pless, Sloane and Ward) of codes over GF(3).

Liebler [24] has found that modular representations of symmetric groups yield very interesting codes. (Naturally, representations of symmetric groups are related to enormous numbers of combinatorial questions (see, e.g., [30])).

Recent results of $\mathrm{E}$. Anders are especially promising: he obtained a coding theoretic proof of the Bruck-Ryser theorem, as well as various generalizations of it-including Hughes' results [19] concerning collineations.

6. Wielandt [39] showed that $|G|<24^{n}$ for a uniprimitive permutation group $G$ of degree $n$. This has just been greatly improved by Babai [1], who proved that

$$
|G|<\exp \left(4 n^{1 / 2}(\log n)^{2}\right)
$$

His proof is entirely combinatorial. Namely, he showed that any primitive coherent configuration on $n$ points has a "distinguishing subset" $S$ of size $<4 n^{1 / 2} \log n$ : for any distinct points $x, y$, there is an $s$ in $S$ such that $(x, s)$ and $(y, s)$ are in different relations. His original motivation for finding such an $S$ came from a problem concerning computational complexity. His methods probably will have other group theoretic applications.

Cameron has pointed out that the classification of all finite simple groups will yield the inequality $|G|<n^{\operatorname{cog} \log n}$ except for some explicit, familiar types of primitive groups. A direct proof of this would undoubtedly have to be partly combinatorial.

An entirely different type of inequality relates the rank of $G$ to $F^{*}(G)$, when the latter group is simple [2], [27], [21]. Complete determinations have been 
made for rank 2, and for rank 3 when $F^{*}(G)$ is an alternating or classical group [2], [11], [23]. The rank 3 representations of the remaining Chevalley groups have yet to be determined; however, this is known to be a finite problem [27]. The determination of all primitive rank 3 groups with $F^{*}(G)$ neither solvable nor simple remains open; combinatorial arguments will presumably be needed in order to relate the underlying graph to the blocks of imprimitivity of $F^{*}(G)$.

\section{REFERENCES}

1. L. Babai, On the order of uniprimitive permutation groups (to appear).

2. E. Bannai, Maximal subgroups of low rank of finite symmetric and alternating groups, J. Fac. Sci. Univ. Tokyo 18 (1972), 475-486.

3. F. Buekenhout and C. Lefèvre, Generalized quadrangles in projective spaces, Arch. Math. 25 (1974), 540-552.

4. P. J. Cameron, Suborbits in transitive permutation groups, Combinatorics, M. Hall, Jr. and J. H. van Lint (eds.), Reidel, Dordrecht, 1975, pp. 419-450.

5. P. J. Cameron, J. M. Goethals and J. J. Seidel, The Krein condition, spherical designs, Norton algebras and permutation groups, Nederl. Akad. Wetensch. A81 (1978), 196-206.

6. P. J. Cameron and W. M. Kantor, 2-transitive and antiflag transitive collineation groups of finite projective spaces, J. Algebra 60 (1979), 384-422.

7. B. N. Cooperstein, Minimal degree for a permutation representation of a classical group, Israel J. Math. 30 (1978), 213-235.

8. __ The geometry of root subgroups in exceptional groups. I, II (to appear).

9. ___ Subgroups of exceptional groups of Lie type generated by long root elements. I, II (to appear).

10. C. W. Curtis, Homology representations of finite groups (to appear).

11. C. W. Curtis, W. M. Kantor and G. M. Seitz, The 2-transitive permutation representations of the finite Chevalley groups, Trans. Amer. Math. Soc. 218 (1976), 1-57.

12. P. Delsarte, An algebraic approach to the association schemes of coding theory, Philips Res. Rep. Suppl. 10 (1973).

13. , Association schemes and t-designs in regular semilattices, J. Combinatorial Theory 20 (1976), 230-243.

14. W. Feit and G. Higman, The nonexistence of certain generalized polygons, J. Algebra 1 (1964), 114-131.

15. W. Haemers, Eigenvalue techniques in design and graph theory, Math. Centrum Amsterdam, 1979.

16. D. G. Higman, Partial geometries, generalized quadrangles and strongly regular graphs, Atti Conv. Geom. Appl., Perugia 1971, pp. 263-293.

17. __ Invariant relations, coherent configurations and generalized polygons, Combinatorics, $\mathbf{M}$. Hall, Jr. and J. H. van Lint (eds.), Reidel, Dordrecht, 1975, pp. 27-43.

18. P. N. Hoefsmit, Representations of Hecke algebras of finite groups with BN-pairs of classical type, $\mathrm{Ph} . \mathrm{D}$. Thesis, Univ. of British Columbia, 1974.

19. D. R. Hughes, Collineations and generalized incidence matrices, Trans. Amer. Math. Soc. 86 (1957), 284-296.

20. W. M. Kantor, Subgroups of classical groups generated by long root elements, Trans. Amer. Math. Soc. 248 (1979), 347-379.

21. (, Permutation representations of the finite classical groups of small degree or rank, J. Algebra 60 (1979), 158-168.

22. $\ldots$ Generalized quadrangles associated with $G_{2}(q)$ (to appear).

23. W. M. Kantor and R. A. Liebler, The rank 3 permutation representations of the finite classical groups (to appear).

24. R. A. Liebler, On codes in the natural representations of the symmetric group (to appear).

25. F. J. MacWilliams and N. J. A. Sloane, The theory of error-correcting codes, North-Holland, Amsterdam, 1977. 
26. M. A. Ronan, A geometric characterization of Moufang hexagons, Ph.D. Thesis, Univ. of Oregon, 1978.

27. G. M. Seitz, Small rank permutation representations of finite Chevalley groups, J. Algebra 28 (1974), 508-517.

28. N. J. A. Sloane, An introduction to association schemes and coding theory, Theory and Application of Special Functions, Academic Press, New York, 1975, pp. 225-260.

29. , Error-correcting codes and invariant theory: New applications of a nineteenth-century technique, Amer. Math. Monthly 84 (1977), 82-107.

30. R. Stanley, Invariants of finite groups, Bull. Amer. Math. Soc. (N.S.) 1 (1979), 475-511.

31. D. Stanton, Some q-Krawtchouk polynomials on Chevalley groups (to appear).

32 Some Erdös-Ko-Rado theorems for Chevalley groups (to appear).

33. J. A. Thas, On generalized quadrangles with parameters $s=q^{2}$ and $t=q^{3}$, Geom. Dedicata 5 (1976), 485-496.

34. Combinatorial characterizations of the classical generalized quadrangles, Geom. Dedicata 6 (1977), 339-351.

35. J. Tits, Sur la géométrie des R-espaces, J. Math. Pures Appl. 36 (1957), 17-38.

36 Les "formes réelles" des groupes de type $E_{6}$, Séminaire Bourbaki No. 162, 1958.

37. A. Wagner, Determination of the finite primitive reflection groups over an arbitrary field of characteristic not two. I, II, III (to appear).

38. H. N. Ward, Quadratic residue codes and symplectic groups, J. Algebra 29 (1974), 150-170.

39. H. Wielandt, Permutation groups through invariant functions and invariant relations, Lecture Notes, Ohio State Univ., 1969.

40. A. Yanushka, Generalized hexagons of order ( $t, t$, Israel J. Math. 23 (1976), 309-324. 